\title{
Providers tried to help patients and families make realistic end of life decisions
}

Norton SA, Bowers BJ. Working toward consensus: providers'strategies to shift patients from curative to palliative

treatment choices. Res Nurs Health 2001 Aug;24:258-69.

\section{QUESTION: What are the strategies used by providers to assist terminally ill patients and their families in shifting from curative to palliative treatment choices?}

Design

Grounded theory.

Setting

Madison, Wisconsin, USA.

\section{Participants}

10 nurses and 5 physicians (12 women and 3 men) who were working in the practice areas of home health and family practice, oncology, and intensive care.

\section{Methods}

Each provider was interviewed once, and 3 providers were interviewed a second time. The interviews were 30-90 minutes in length, and were tape recorded and transcribed verbatim. Analysis was done after each interview and the questions in subsequent interviews became increasingly focused on evolving categories. Theoretical sampling was built into the interviews with questions designed to determine the types of strategies providers use to assist patients to understand their situations and make realistic decisions.

\section{Main findings}

Providers' strategies aimed to help patients achieve a good death (eg, comfortable and peaceful) and avoid a bad one (eg, unnecessarily painful with overly aggressive treatment). The providers' main challenge was getting patients and their families to shift their goals and decisions to realistic ones in the context of a terminal illness. Providers described laying the groundwork so that patients and their families would have the background necessary to make realistic decisions. This was achieved by teaching about prognosis and treatment choices and discussing the possibility of death (planting seeds). Shifting the picture involved helping patients and their families move from unrealistic curative decisions to realistic palliative ones. Providers described the importance of communicating with each other and with patients and families to ensure that everyone had the same information and were working towards a common goal $-\mathrm{a}$ good death. Family meetings, including conference calls to out of town family members, provided the mechanism for such communication. Shifting the picture was also achieved by changing the scope of choice by introducing new, realistic choices to patients; changing the value of treatment options so that patient comfort was emphasised over cure; and changing indicators so that patients and their families could correctly monitor the patient's condition. Once patients and families had shifted the picture, providers reinforced the patients' acceptance of the new picture by involving social workers, clergy, or palliative care services; redirecting hope to more realistic goals; and by repeating and reiterating information.

\section{Conclusion}

Providers assisted terminally ill patients and their families in the transition from unrealistic curative goals to realistic palliative goals by laying the groundwork, shifting the picture, and reinforcing acceptance of the new picture.

\section{COMMENTARY}

The study by Norton and Bowers provides helpful information for healthcare professionals who care for patients at the end of life. The researchers have developed a model for healthcare professionals designed to help patients and families achieve a realistic approach to end of life situations, and facilitate a transition from a curative to a palliative perspective. Unless a palliative approach to end of life decisions is used, many opportunities to assist patients and family members go unnoticed and unfulfilled.

The study, using a grounded theory approach, revealed the concepts of end of life care articulated in interviews with healthcare professionals. The researchers organised those concepts and, based on interview data, discerned the relationships among those concepts to identify a formal model of the process used by healthcare providers of end of life patients. The study also revealed some pragmatic strategies in facilitating this process for an outcome of a "good death".

Although the study included 5 family members initially, only the data obtained from the nurses and physicians were included in the analysis. Hence, the model was derived solely from information gathered from healthcare professionals and is dependent on their inferences and judgments. Because client and family perceptions are crucial to understanding what constitutes a "good death", the derivation of the model is open to criticism. The model warrants careful scrutiny by all participants involved in the end of life process. Rigorous evaluation designed to test this model is needed.

The study offers a model for helping healthcare professionals understand and facilitate a process to help end of life patients and their families make a transition from unrealistic expectations to realistic treatment choices and successful end of life care.

Stephen D Krau, RN, PhD Professor and Coordinator, Continuing Education School of Nursing Middle Tennessee State University Murfreesboro, Tennessee, USA

1 Byock IR. The nature of suffering and the nature of opportunity at the end of life. Clin Geriatr Med 1996;12:237-52. 